\title{
Modelagem e simulação do processo de secagem das cascas de jabuticaba (Myrciaria cauliflora)
}

\author{
Modeling and simulation of the drying process of jabuticaba shells (Myrciaria cauliflora) \\ Modelado y simulación del proceso de secado de cáscaras de jabuticaba (Myrciaria cauliflora)
}

Recebido: 22/02/2021 | Revisado: 28/02/2021 | Aceito: 05/03/2021 | Publicado: 14/03/2021

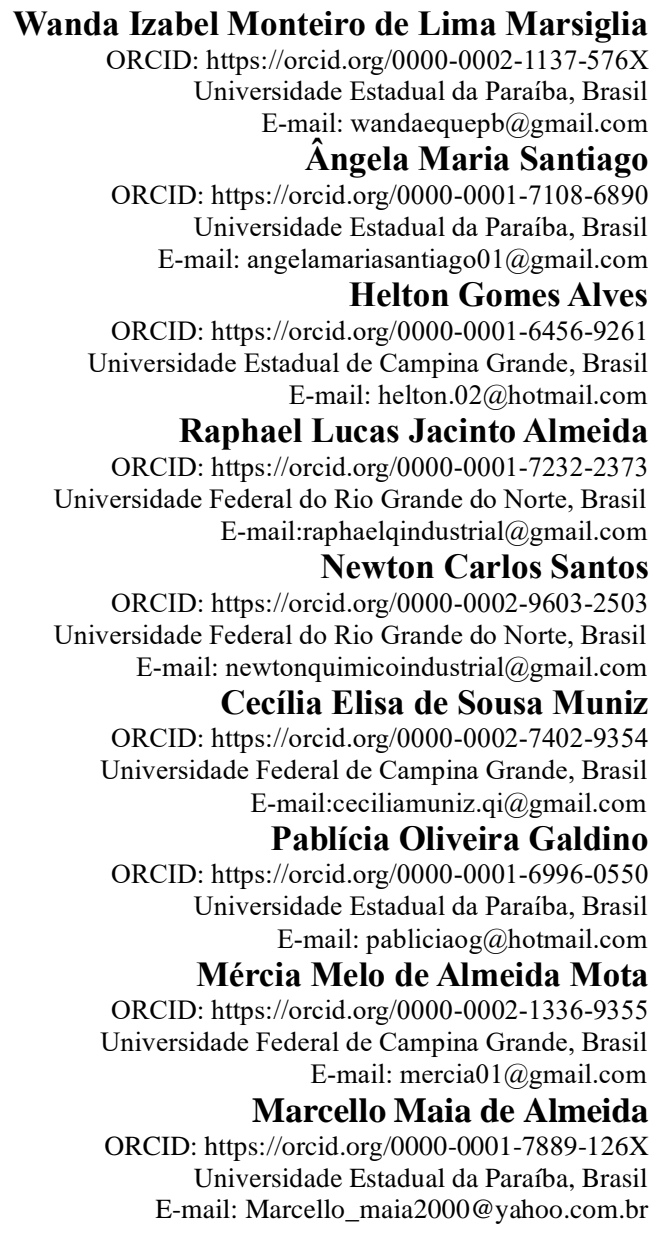

\section{Resumo}

O presente trabalho tem como objetivo determinar a composição físico-química e dos compostos bioativos da casca de jabuticaba fresca, realizar a sua cinética de secagem em três diferentes temperaturas de ar de secagem, ajustar modelos matemáticos empíricos e difusivo aos dados experimentais, produzir o pó e avaliar efeito da temperatura de secagem sobre a sua composição. Os frutos foram colhidos, lavados e higienizados com solução de hipoclorito de sódio, em seguida os frutos foram despolpados manualmente e as frações sólidas, polpa, cascas e sementes foram separadas. As cascas de jabuticaba foram secas em estufa com circulação de ar nas temperaturas de 45,50 e $55^{\circ} \mathrm{C}$ e velocidade de 1.0 $\mathrm{m} / \mathrm{s}$. Foram construídas as curvas da cinética de secagem, representadas pela razão do teor de água em função do tempo de secagem em minutos, ajustando aos modelos matemáticos de Handerson e Pabis, Logarítmico, Midilli, Page e Newton aos dados experimentais. Os modelos foram selecionados tomando-se como parâmetro a magnitude do coeficiente de determinação $\left(\mathrm{R}^{2}\right)$ e a função qui-quadrado $\left(\chi^{2}\right)$. Logo após a secagem as cascas foram trituradas para obtenção do pó, o qual foi caracterizado antes e após a secagem quanto as características físicas, químicas, compostos bioativos e atividade antioxidante. As cascas frescas de jabuticaba apresentaram altos teores de compostos fenólicos totais e taninos totais. O modelo de Midilli se destacou dos demais por apresentar o maior valor de R2 (>99\%) e o menor valor da função qui-quadrado $(<0.000327)$ para as três temperaturas estudadas, em especial para a de $45^{\circ} \mathrm{C}$. A cinética de secagem das cascas de jabuticaba mostrou que a temperatura influenciou no processo, e que a constante " $k$ " aumentou 
com a sua elevação, e o tempo do processo diminuiu em função do seu aumento. A temperatura de $45^{\circ} \mathrm{C}$ garantiu uma maior preservação dos compostos fenólicos totais, antocianinas totais e flavonoides totais do pó das cascas da jabuticaba, podendo ser uma alternativa como ingrediente na elaboração de vários produtos dentre eles: pão, bolo, biscoito, iogurte e bebida láctea por ter uma quantidade significativa desses compostos bioativos.

Palavras-chave: Alimento funcional; Compostos bioativos; Conservação; Resíduo agroindustrial.

\begin{abstract}
The present work aims to determine the physical-chemical composition and bioactive compounds of the fresh jabuticaba bark, perform its drying kinetics at three different drying air temperatures, adjust empirical and diffusive mathematical models to the experimental data, produce the powder and evaluate the effect of drying temperature on its composition. The fruits were harvested, washed and sanitized with sodium hypochlorite solution, then the fruits were manually pulped and the solid fractions, pulp, peels and seeds were separated. The jabuticaba peels were dried in an oven with air circulation at temperatures of 45,50 and $55^{\circ} \mathrm{C}$ and a speed of $1.0 \mathrm{~m} / \mathrm{s}$. Curves of drying kinetics were constructed, represented by the ratio of water content to drying time in minutes, adjusting to the mathematical models of Handerson and Pabis, Logarithmic, Midilli, Page and Newton to the experimental data. The models were selected taking as a parameter the magnitude of the determination coefficient $\left(\mathrm{R}^{2}\right)$ and the chi-square function $\left(\chi^{2}\right)$. Shortly after drying, the shells were crushed to obtain the powder, which was characterized before and after drying in terms of physical, chemical, bioactive compounds and antioxidant activity. Fresh jabuticaba peels showed high levels of total phenolic compounds and total tannins. The Midilli model stood out from the others for presenting the highest value of R2 (> $99 \%)$ and the lowest value of the chi-square function $(<0.000327)$ for the three temperatures studied, especially for the $45^{\circ} \mathrm{C}$. The drying kinetics of jabuticaba peels showed that the temperature influenced the process, and that the constant " $\mathrm{k}$ " increased with its elevation, and the time of the process decreased as a result of its increase. The temperature of $45^{\circ}$ $\mathrm{C}$ ensured a greater preservation of the total phenolic compounds, total anthocyanins and total flavonoids of the powder of the jabuticaba peels, and can be an alternative as an ingredient in the elaboration of several products, among them: bread, cake, biscuit, yogurt and drink milk because it has a significant amount of these bioactive compounds.
\end{abstract}

Keywords: Functional food; Bioactive compounds; Conservation; Agro-industrial waste.

\title{
Resumen
}

El presente trabajo tiene como objetivo determinar la composición físico-química y compuestos bioactivos de la corteza fresca de jabuticaba, realizar su cinética de secado a tres temperaturas de aire de secado diferentes, ajustar modelos matemáticos empíricos y difusivos a los datos experimentales, producir el polvo y evaluar el efecto de temperatura de secado en su composición. Los frutos se recolectaron, lavaron y desinfectaron con solución de hipoclorito de sodio, luego los frutos se despulparon manualmente y se separaron las fracciones sólidas, pulpa, cáscaras y semillas. Las cáscaras de jabuticaba se secaron en un horno con circulación de aire a temperaturas de 45,50 y $55^{\circ} \mathrm{C}$ y una velocidad de $1,0 \mathrm{~m} / \mathrm{s}$. Se construyeron curvas de cinética de secado, representadas por la relación entre el contenido de agua y el tiempo de secado en minutos, ajustándose a los modelos matemáticos de Handerson y Pabis, Logarithmic, Midilli, Page y Newton a los datos experimentales. Los modelos fueron seleccionados tomando como parámetro la magnitud del coeficiente de determinación $\left(\mathrm{R}^{2}\right)$ y la función chi-cuadrado $\left(\chi^{2}\right)$. Poco después del secado, las cáscaras se trituraron para obtener el polvo, que se caracterizó antes y después del secado en términos de compuestos físicos, químicos, bioactivos y actividad antioxidante. Las cáscaras frescas de jabuticaba mostraron altos niveles de compuestos fenólicos totales y taninos totales. El modelo de Midilli se destacó de los demás por presentar el mayor valor de R2 (> 99\%) y el menor valor de la función chi-cuadrado $(<0.000327)$ para las tres temperaturas estudiadas, especialmente para los 45 ${ }^{\circ} \mathrm{C}$. La cinética de secado de las cáscaras de jabuticaba mostró que la temperatura influyó en el proceso y que la constante " $\mathrm{k}$ " aumentó con su elevación y el tiempo del proceso disminuyó como resultado de su aumento. La temperatura de $45{ }^{\circ} \mathrm{C}$ aseguró una mayor conservación de los compuestos fenólicos totales, antocianinas totales y flavonoides totales del polvo de las cáscaras de jabuticaba, pudiendo ser una alternativa como ingrediente en la elaboración de varios productos, entre ellos: pan, torta, bizcocho, yogur y leche de bebida porque tiene una cantidad significativa de estos compuestos bioactivos.

Palabras clave: Comida funcional; Compuestos bioactivos; Conservación; Residuos agroindustriales.

\section{Introdução}

Os subprodutos industriais de frutas e vegetais são constituídos principalmente por: cascas, frações de bagaço e sementes, que podem ser uma boa fonte de compostos bioativos, além de conterem na sua composição carboidratos, fibras alimentares, compostos aromatizantes e fitoquímicos (Coman et al., 2019). Normalmente as cascas são descartadas, correspondendo cerca de $30 \%$ a $43 \%$ do fruto, gerando grandes quantidades de resíduos e provocando impacto ambiental devido 
ao lançamento excessivo no meio ambiente (Alezandro et al., 2013). A polpa da jabuticaba é utilizada pela indústria alimentícia na fabricação de sucos, geleias, sorvetes, vinagres, bebidas alcoólicas fermentadas e destiladas (Wu et al. 2013).

Foi demonstrado que o pó de casca de jabuticaba (PCJ) tem fortes efeitos antioxidantes, anti-inflamatórios, e antidiabéticos em ratos obesos (Batista et al. 2018). Além disso, o PCJ foi recentemente nomeado entre as superfrutas devido aos seus relevantes efeitos de promoção da saúde e demonstrou reduzir os níveis de glicose e insulina pós-prandial e aumentar a capacidade antioxidante em humanos (Chang et al., 2018). Esses efeitos do PCJ foram atribuídos apenas a compostos fenólicos livres que são facilmente extraídos por solventes orgânicos (Quatrin et al., 2019).

Segundo Pádua et al. (2017) uma alternativa para aproveitamento dos resíduos da jabuticaba é a elaboração do pó, que pode ser utilizado para o preparo e enriquecimento de alimentos e bebidas. Para obtenção do pó se faz necessário utilizar a técnica de secagem considerando o binômio tempo e temperatura, visto que os compostos fenólicos podem ser degradados durante o processo, permitindo assim minimizar as possíveis alterações causadas pelas reações químicas, enzimáticas e microbiológicas (Gastaldi et al., 2018). A fim de indicar o potencial de aproveitamento deste resíduo na elaboração de novos produtos alimentícios, contribuir para a sua agregação de valor como matéria-prima e auxiliar na redução dos custos de produção. O presente trabalho tem como objetivo determinar a composição físico-química e dos compostos bioativos da casca de jabuticaba fresca, realizar a sua cinética de secagem em três diferentes temperaturas de ar de secagem, ajustar modelos matemáticos empíricos e difusivo aos dados experimentais, produzir o pó e avaliar efeito da temperatura de secagem sobre a sua composição.

\section{Metodologia}

\subsection{Obtenção dos frutos e preparação de cascas frescas}

A jabuticaba da variedade Sabará Myrciaria cauliflora (Mart. O. Berg) foi obtida na feira livre da cidade de Alagoa Nova, Paraíba, Brasil. Os frutos foram colhidos manualmente, lavados e higienizados com solução de hipoclorito de sódio (Neon Comercial, São Paulo, Brasil) diluída em água destilada para obtenção de solução de cloro livre $2.5 \%$. A jabuticaba foi despolpada manualmente e as frações sólidas, polpa, cascas e sementes foram separadas.

\subsection{Cinética de secagem}

As cascas de jabuticaba foram colocadas em telas de aço inoxidável, e uniformemente espalhadas. Para iniciar o experimento, as bandejas foram colocadas em um secador com circulação de ar (fabricante MARCONI, modelo MA048) com velocidade de ar de $1.0 \mathrm{~m} \mathrm{~s}^{-1}$, para a realização das cinéticas de secagem nas temperaturas de 45,50 e $55^{\circ} \mathrm{C}$, no início e no final das secagens foram determinadas as massas secas e calculados os teores de água de acordo com Brasil (2008).

Através dos dados experimentais, foi possível calcular os valores da razão do teor de água (Equação 1).

$$
X^{*}=\frac{X_{b s}-X_{e}}{X_{b s(\text { inicial })}-X_{e}}
$$

Em que: $\mathrm{X}^{*}$ é a razão de umidade (adimensional); $\mathrm{X}_{\mathrm{e}}$ é o teor de água de equilíbrio em base seca; $\mathrm{X}_{\mathrm{bs}}$ é o teor de água em base seca; $X_{\text {bs (inicial) }}$ é o teor de água inicial em base seca.

Com o cálculo da razão de teor de água das cascas de jabuticaba, traçaram-se as curvas da cinética de secagem, representada pela razão do teor de água em função do tempo de secagem em minutos, ajustando os modelos matemáticos de 
Handerson e Pabis (Equação 2), Logarítmico (Equação 3), Midilli (Equação 4), Page (Equação 5) e Newton (Equação 6 ) aos dados experimentais.

$$
\begin{aligned}
& X^{*}=a e^{-k t} \\
& X^{*}=a e^{-k t}+c \\
& X^{*}=a e^{-k t^{n}}+b t \\
& X^{*}=e^{-k t^{n}} \\
& X^{*}=e^{-k t}
\end{aligned}
$$

Em que: $X$ "é a razão de umidade (admensional); k é a constante de secagem; "a", "b", "n" e "c" são os parâmetros das equações; té o tempo de secagem (min).

Para análise dos ajustes dos modelos matemáticos aos dados experimentais, foi utilizado o programa computacional Statistica, versão 7, utilizando-se a análise de regressão não-linear, pelo método Quasi-Newton. Os modelos foram selecionados tomando-se como parâmetro a magnitude do coeficiente de determinação $\left(\mathrm{R}^{2}\right)$ e a função qui-quadrado $\left(\chi^{2}\right)$ (Equação 7$)$.

$$
\chi^{2}=\frac{\sum_{i=1}^{N}\left(X_{\mathrm{exp}, i}^{*}-X_{p r e, i}^{*}\right)^{2}}{N-n}
$$

Em que: $\chi^{2}$ é a função qui-quadrado; $X_{\text {exp, } i}^{*}$ é a razaõ de umidade experimental; $X_{\text {pre }, i}^{*}$ é a razão de umidade prevista pelo modelo; $\mathrm{N}$ é o número de dados experimentais; $\mathrm{n}$ é o número de coeficientes e constantes do modelo.

\subsection{Solução analítica da equação de difusão}

O teor médio de umidade do sólido com geometria de parede infinita no momento t é dado por (Luivok, 1968), desconsiderando-se a contração volumétrica é dado pela Equação (8)

$$
X^{*}(t)=\sum_{n=1}^{16} B_{n} \exp \left(-\mu_{n}^{2} \frac{D e f}{(L / 2)^{2}} t\right)
$$

Em que: $X^{*}(t)$ é a razão de umidade no instante $t$; $L$ é a espessura; Defé a difusividade; t é o tempo.

Os parâmetros da Equação 9, foram obtidos através das Equações 9,10 e 11:

$$
B_{n}=\frac{2 B i^{2}}{\mu_{n}^{2}\left(B i^{2}+B i+\mu_{n}^{2}\right)}
$$




$$
B i=\frac{h(L / 2)}{D e f}
$$

Em que: h é o coeficiente convectivo de transferência de calor; Bi é o número de Biot.

$$
\cot \mu=\frac{\mu}{B i}
$$

Em que: a Equação 12 é caracteristica para a parede infinita. Para obtenção da solução analítica da equação de difusão a otimização do processo foi feita de acordo com as metodologias propostas por Silva et al. (2010a), utilizando o software Convective Adsorption-Dessorption.

Uma vez que os parâmetros do processo para o modelo de difusão foram determinados, a distribuição de umidade em um dado momento $t$ foi determinado pela solução da equação de difusão dependente da posição x, com a origem estabelecida no centro da espessura das cascas conforme a Equação 12:

$$
X^{*}(x, t)=\sum_{n=1}^{n t} A_{n} \cos \left(\frac{\mu_{n}}{L / 2} x\right) \exp \left[-\frac{\mu_{n}^{2}}{(L / 2)^{2}} D_{e f} t\right]
$$

Em que: o parâmetro $A_{n}$ é dado pela Equação 13:

$$
A_{n}=\frac{4 \sin \mu_{n}}{2 \mu_{n}+\sin \left(2 \mu_{n}\right)}
$$

\subsection{Rendimento das cascas secas}

O rendimento da casca seca da jabuticaba em relação ao fruto inteiro foi determinado pela Equação 14.

Rendimento $(\%)=\frac{m u-m c s}{m u} * 100$

Em que: $m и$ é a massa da casca úmida (g); $m c s$ é a massa de casca seca (g).

\subsection{Obtenção do pó da casca de jabuticaba}

Após a secagem, as cascas foram moídas em moinho de facas (fabricante TECNAL). As cascas secas e moídas foram denominadas pó de jabuticaba, nas quais foram acondicionadas em embalagem a vácuo, laminadas e compostas por duas camadas de PET-met (Poliéster - tereftalato de polietileno metalizado) com espessura de $12 \mu \mathrm{m}$, identificadas nas respectivas temperaturas de secagem e armazenado em temperatura ambiente protegido da luz. 


\subsection{Caracterização físico-química}

A atividade de água $(\mathrm{a} \omega)$ foi determinada usando o dispositivo Decagon ${ }^{\circledR}$ Aqualab $\mathrm{CX}-2 \mathrm{~T}$ a $25^{\circ} \mathrm{C}$, o teor de água foi determinado pelo método gravimétrico (A.O.A.C., 2016). A coloração foi realizada com o colorímetro digital CR 300 (Minolta, New Jersey, EUA), determinada de acordo com o CIE-L $* a * b *$.

\subsection{Compostos bioativos e atividade antioxidante}

Os compostos fenólicos totais foram quantificados pelo método de Folin-Ciocalteau descrito por Waterhouse (2006), usando ácido gálico como padrão. Os cálculos realizados para a determinação dos compostos fenólicos foram baseados em uma curva padrão com ácido gálico, e as leituras foram realizadas em espectrofotômetro a $765 \mathrm{~nm}$, com resultados expressos em (mg $100 \mathrm{~g}^{-1}$ ) de ácido gálico. Os teores de antocianinas e de flavonoides foram determinados seguindo o método de $\mathrm{pH}$ único descrito por Francis (1982). Os taninos totais foram determinados seguindo metodologia (Pansera et al. 2003). A atividade antioxidante por DPPH foi realizada utilizando a metodologia descrita por (Rufino et al. 2010), com adaptações. A atividade antioxidante foi determinada pelo método $\mathrm{ABTS}^{++}$utilizando o método proposto por (Re et al. 1999), com modificações feitas por (Rufino et al. 2007). Para ambas as análises, água destilada foi utilizada como solvente extrativo.

\subsection{Tratamento Estatístico}

Todos os experimentos foram executados em triplicata, e os resultados foram submetidos ao teste de Tukey, adotandose o nível de 5\% de significância. Todas as análises estatísticas foram desenvolvidas usando o software STATISTICA versão 7.0 (Stasoft, 2007).

\section{Resultados e Discussão}

\subsection{Caracterização das cascas de jabuticaba frescas}

Os resultados obtidos da caracterização física, dos compostos bioativos e atividade antioxidante da casca de jabuticaba frescas estão expressos na Tabela 1. 
Tabela 1. Caracterização físico-química, dos compostos bioativos e atividade antioxidante da casca de jabuticaba fresca

\begin{tabular}{|c|c|}
\hline Parâmetros & $\begin{array}{c}\text { Valores médios } \pm \\
\text { DP }\end{array}$ \\
\hline Atividade de água $\left(a_{w}\right)$ & $0.98 \pm 0.02$ \\
\hline Teor de água \% (b.u.) & $82.93 \pm 1.79$ \\
\hline Luminosidade $(* \mathbf{L})$ & $13.71 \pm 0.01$ \\
\hline Intensidade de vermelho $\left({ }^{*} a\right)$ & $6.71 \pm 0.01$ \\
\hline Intensidade de azul $(* \mathbf{b})$ & $4.18 \pm 0.02$ \\
\hline Compostos fenólicos totais (mg GAE $\mathbf{1 0 0 g}^{-1}$ ) & $958.22 \pm 5.56$ \\
\hline Antocianinas totais $\left(\mathrm{mg}^{100 \mathrm{~g}^{-1}}\right)$ & $29.66 \pm 0.50$ \\
\hline Flavonoides totais $\left(\mathrm{mg}^{100 \mathrm{~g}^{-1}}\right)$ & $67.29 \pm 0.53$ \\
\hline Taninos totais (mg ácido tânico $100 \mathrm{~g}^{-1}$ ) & $477.01 \pm 4.13$ \\
\hline Atividade antioxidante por DPPH (\%) & $37.38 \pm 2.58$ \\
\hline Atividade antioxidante por $\operatorname{ABTS}^{++}\left(\mu \mathrm{M}\right.$ TEAC $\left.\mathrm{g}^{-1}\right)$ & $394.44 \pm 5.37$ \\
\hline
\end{tabular}

Nota: GAE (Equivalente de Ácido Gálico), TEAC - Capacidade antioxidante em equivalente de Trolox. Fonte: Autores.

Observa-se que o teor de água da casca de jabuticaba in natura apresentou valor acima de $80 \%$ devido à grande quantidade de água presente, sendo similar ao encontrado por Lima et al. (2008) quando caracterizaram os frutos de jabuticaba e obtiveram frações de $84.24 \%$ de água na casca da variedade Sabará. Wu et al. (2013) estudaram os benefícios dos fitoquímicos presentes na casca de jabuticaba e obtiveram o valor de $87.10 \%$ para o teor de água. Lenquiste et al. (2015) registraram valores superiores $(86.12 \%)$ para a casca de jabuticaba.

Analisando o parâmetro de cor, foi possível observar que a coloração da casca em relação a luminosidade foi de 13.71, indicando que a amostra apresenta baixa luminosidade. As cromaticidades expressas por ( $\left.\mathrm{a}^{*}\right)$ e ( $\mathrm{b}^{*}$ ) foram de 6.71 e 4.18 , respectivamente, onde existe a tendência para a cor vermelha e azul, definindo assim visualmente a cor da casca de jabuticaba, como preta azulada, isso foi contestado por Abe et al. (2012) no qual definiram como púrpura. Curi et al. (2018) encontraram valores de $\mathrm{L}^{*} 12.53$ e as cromaticidades de $\mathrm{a}^{*} 6.71$ e b* 4.03, valores próximos ao encontrado no presente estudo. A análise de cor está relacionada com o teor em antocianinas, sendo a maior presença na casca do fruto.

Observa-se que os valores médios de compostos fenólicos totais foram próximos ao encontrado por Dessimoni-Pinto et al. (2011) (1006.44 mg GAE $100 \mathrm{~g}^{-1}$ ) e inferiores aos reportados por Seraglio et al. (2018) que encontraram (1443.63 mg GAE $100 \mathrm{~g}^{-1}$ ). Os resultados obtidos neste estudo demonstraram que a casca de jabuticaba apresenta alto teor de compostos fenólicos, entretanto, a diferença nas quantidades obtidas de fenólicos pode estar relacionada com as variedades, região, solo e clima, bem como a metodologia e os solventes utilizados (Morales-Soto et al., 2014).

Ao quantificar as antocianinas totais, observou-se que o valor médio obtido neste estudo está na faixa reportada por Alezandro et al. (2013) que observaram variação de 4.90 a $147.00 \mathrm{mg} 100 \mathrm{~g}^{-1}$, o aumento da concentração deve-se ao estádio de

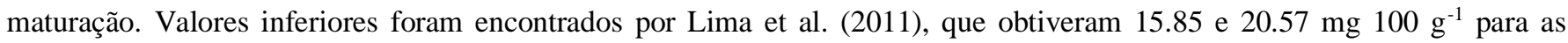
variedades Paulista e Sabará, respectivamente. Com relação aos flavonoides, verificou-se que o conteúdo obtido neste estudo foi inferior ao reportado por Silva et al. (2014) ao quantificar os compostos bioativos em subprodutos de frutas tropicais do Brasil, encontraram para a casca de cereja (207.87 mg $\left.100 \mathrm{~g}^{-1}\right)$ e para a casca de acerola (98.05 $\left.\mathrm{mg}^{100 \mathrm{~g}^{-1}}\right)$. Para taninos totais observa- 
se que a casca in natura apresentou teor elevado, este resultado provavelmente está associado à adstringência presente na casca de jabuticaba in natura.

Os resultados dos bioativos da casca de jabuticaba frescas da espécie Sabará demonstraram ter alto teor de compostos fenólicos, dentre eles, antocianinas e flavonoides. $\mathrm{O}$ valor encontrado para a atividade antioxidante da casca de jabuticaba fresca utilizando o método $\mathrm{ABTS}^{++}$foi de $394.44 \mu \mathrm{mol}$ Trolox g${ }^{-1}$. Rufino et al. (2010) encontraram valor de atividade antioxidante no camu camu de $393.43 \mu \mathrm{mol}$ Trolox $\mathrm{g}^{-1}$.

Na Tabela 2, estão apresentados os valores obtidos para os parâmetros das equações dos modelos empíricos de Handerson \& Pabis, Logarítmico, Midilli, Page e Newton ajustados aos dados experimentais da cinética de secagem das cascas de jabuticaba nas temperaturas de 45,50 e $55^{\circ} \mathrm{C}$.

Tabela 2. Parâmetros matemáticos e estatísticos obtidos pelos ajuste dos modelos matemáticos empíricos aos dados experimentais da cinética de secagem das cascas de jabuticaba.

\begin{tabular}{|c|c|c|c|c|c|c|c|}
\hline \multirow{2}{*}{ Modelos } & \multirow{2}{*}{$\mathbf{T}\left({ }^{\circ} \mathrm{C}\right)$} & \multicolumn{4}{|c|}{ Parâmetros } & \multirow{2}{*}{$\mathbf{R}^{2}$} & \multirow{2}{*}{$\chi^{2}$} \\
\hline & & $\overline{\mathbf{A}}$ & $\mathbf{K}$ & $\mathrm{c} / \mathrm{n}$ & b & & \\
\hline \multirow{4}{*}{$\begin{array}{c}\text { Handerson \& } \\
\text { Pabis }\end{array}$} & 45 & 0.965897 & 0.001998 & - & - & 0.9946 & 0.035278 \\
\hline & 50 & 0.943530 & 0.002959 & - & - & 0.9986 & 0.002890 \\
\hline & 55 & 0.972648 & 0.004895 & - & - & 0.9988 & 0.024036 \\
\hline & 45 & 1.108812 & 0.001402 & -0.171426 & - & 0.9977 & 0.002074 \\
\hline \multirow[t]{3}{*}{ Logarítmico } & 50 & 0.940198 & 0.003002 & 0.005029 & - & 0.9986 & 0.000049 \\
\hline & 55 & 0.987056 & 0.004627 & -0.021452 & - & 0.9992 & 0.000080 \\
\hline & 45 & 0.998726 & 0.008404 & 0.715763 & -0.000194 & 0.9993 & 0.000079 \\
\hline \multirow[t]{3}{*}{ Midilli } & 50 & 0.995448 & 0.008604 & 0.815232 & -0.000043 & 0.9990 & 0.000327 \\
\hline & 55 & 0.959587 & 0.008841 & 1.029155 & -0.000016 & 0.9992 & 0.002079 \\
\hline & 45 & - & 0.002612 & 0.964563 & - & 0.9929 & 0.064279 \\
\hline \multirow[t]{3}{*}{ Page } & 50 & - & 0.005755 & 0.976381 & - & 0.9984 & 0.023601 \\
\hline & 55 & - & 0.006742 & 0.870187 & - & 0.9990 & 0.037458 \\
\hline & 45 & - & 0.002612 & 0.964563 & - & 0.9929 & 0.064279 \\
\hline \multirow[t]{2}{*}{ Newton } & 50 & - & 0.005755 & 0.976381 & - & 0.9984 & 0.023601 \\
\hline & 55 & - & 0.006742 & 0.870187 & - & 0.9990 & 0.037458 \\
\hline
\end{tabular}

Fonte: Autores.

Pode-se observar por meio da Tabela 2 que, para todos os modelos matemáticos aplicados a constante de secagem " $k$ " aumentou com o aumento da temperatura. Fato este também observado por Santos et al., (2017a), ao realizarem a cinética de secagem de cascas de pitaya nas temperaturas de 50,60 e $70{ }^{\circ} \mathrm{C}$. O parâmetro "k" tende a aumentar, visto que maiores temperaturas acarretam maiores taxas de secagem chegando ao teor de água de equilíbrio em menor tempo de submissão (Nascimento et al., 2018).

O parâmetro "n" do modelo de Newton apresentou aumento nos seus valores conforme teve o aumento da temperatura de secagem. E os parâmetros "a" e "c" do modelo Logarítmico e Midilli, não apresentaram comportamento definido com incremento da temperatura.

Para todos os modelos aplicados o coeficiente de determinação apresentou valores superiores a $0.99\left(R^{2}>0.99\right)$ entretanto, a avaliação de apenas um único parâmetro estatístico não constitui um bom critério de seleção para modelos não- 
lineares. Silva et al., (2019), mencionaram que para um modelo se ajustar adequadamente aos dados experimentais, é essencial que $\mathrm{R}^{2}$ seja maior que 0.99 e a função qui-quadrado seja o mais baixo possível.

Com relação aos valores obtidos para função qui-quadrado os modelos matemático de Page $\left(45^{\circ} \mathrm{C}\right)$ e de Newton $(45$ ${ }^{\circ} \mathrm{C}$ ) obtiveram os maiores valores de 0.064279 ambos. O modelo Logarítmico $\left(50^{\circ} \mathrm{C}\right)$ e Midilli $\left(45^{\circ} \mathrm{C}\right)$ apresentaram os menores valores dessa função 0.000049 e 0.000079 , respectivamente. Sendo, portanto, o modelo matemático de Midilli o que melhor descreve o processo de secagem das cascas de jabuticaba nas temperaturas de 45,50 e $55^{\circ} \mathrm{C}$. Costa et al., (2016), realizaram a cinética de secagem de cascas de jabuticaba nas temperaturas de 40,50, 60 e $70{ }^{\circ} \mathrm{C}$ e observaram que o modelo de Midilli apresentou melhor ajuste aos dados experimentais.

A Figura 1 descreve a cinética de secagem das cascas de jabuticabas para as três diferentes temperaturas de secagem ajustado ao modelo empírico de Midilli.

Figura 1. Simulação da Cinética de secagem das cascas de jabuticaba usando o modelo empírico Midilli nas temperaturas de 45, 50 e $55^{\circ}$ C. Fonte: Própria (2021).

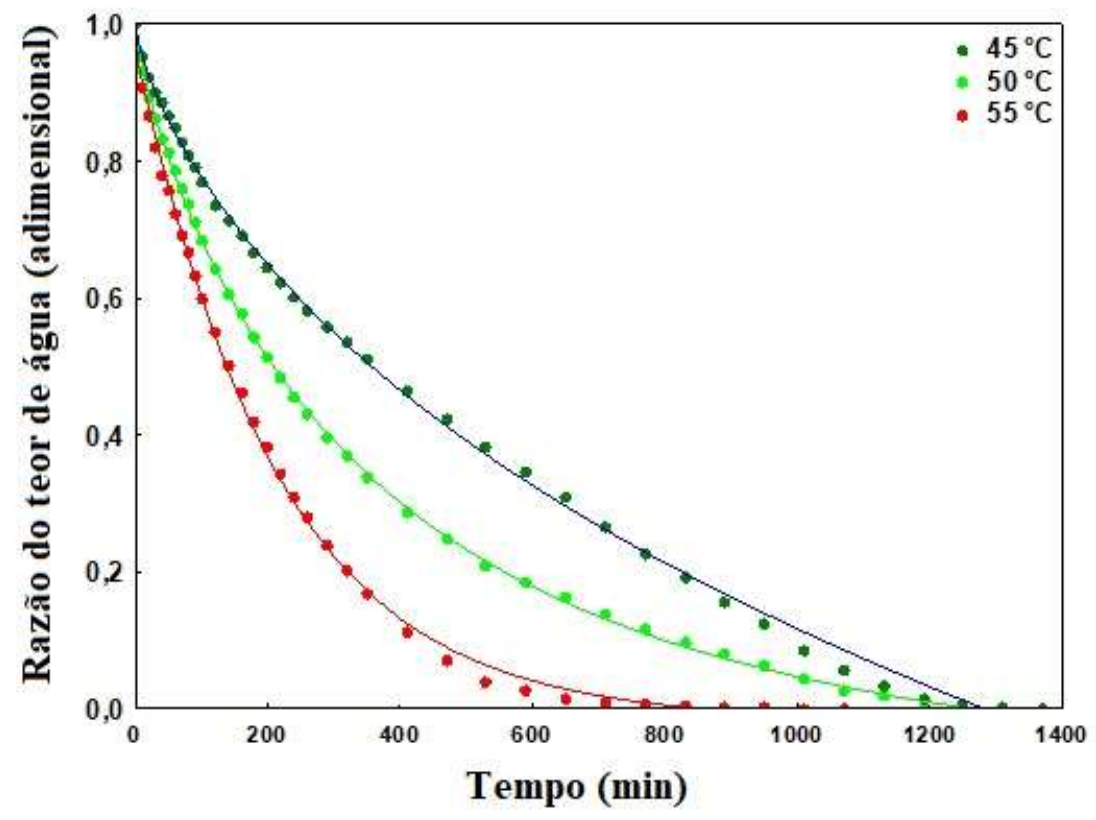

Fonte: Autores.

Pode-se observar que, quando se aumentou a temperatura de secagem, diminuiu os tempos para que as cascas atingisse o equilíbrio, correspondendo a 1310, 1190 e $1010 \mathrm{~min}$, para as respectivas temperaturas 45,50 e $55^{\circ} \mathrm{C}$. Segundo Melo et al., (2015), esse comportamento deve-se ao fato das maiores taxas de remoção de água do produto ocorrerem nas maiores temperaturas, o que reduz o tempo de secagem. Santos et al., (2019a) afirmaram que, apesar do aumento de temperatura reduzir o tempo de secagem, uma condição térmica elevada quanto a exposição excessiva ao calor pode degradar componentes termossenssíveis.

Na Figura 2, observa-se a distribuição espacial de umidade no interior do produto na temperatura de $45^{\circ} \mathrm{C}$ e nos seus diferentes tempos de secagem. 
Figura 2. Distribuição espacial de umidade no interior do produto para temperatura de $45^{\circ} \mathrm{C}$ de 100 a 400 min. Fonte: Própria (2021).

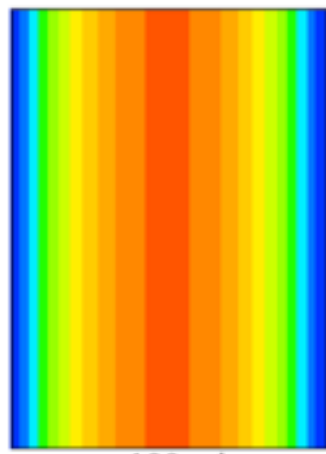

$100 \mathrm{~min}$

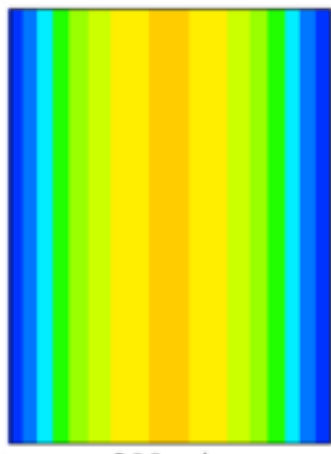

$200 \mathrm{~min}$

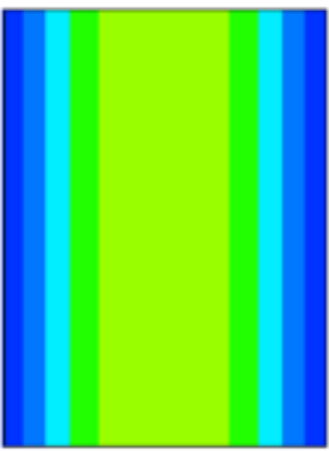

$300 \mathrm{~min}$

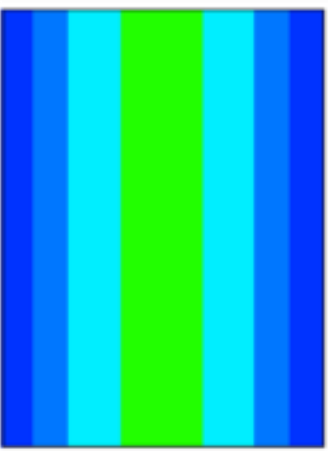

$400 \mathrm{~min}$

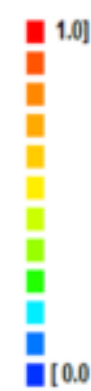

Fonte: Autores.

Através da Figura 2, percebe-se que o teor de umidade adimensional, diminui nas regiões periféricas e aumenta na parte central nos primeiros 400 minutos de secagem, ou seja, o processo de redução do teor de umidade se deu de fora para dentro do produto. E quando se teve aumento no tempo de secagem menores eram as variações dos gradientes de umidade em seu interior. Estão expressos na Tabela 3, os valores obtidos pelo ajuste do modelo de difusão considerando a geometria de parede infinita aos dados experimentais da cinética de secagem das cascas de jabuticaba.

Tabela 3. Resultados obtidos por solução analítica da equação de difusão e para a cinética de secagem das cascas de jabuticaba e caracterizações físicas, físico-químicas e de compostos bioativos dos pós obtidos.

\begin{tabular}{cccc}
\hline Parâmetros & \multicolumn{3}{c}{ Temperaturas } \\
\cline { 2 - 4 } & $\mathbf{4 5}^{\circ} \mathbf{C}$ & $\mathbf{5 0}{ }^{\circ} \mathbf{C}$ & $\mathbf{5 5}^{\circ} \mathbf{C}$ \\
\hline Def $\mathbf{x} \mathbf{1 0}^{-11}$ & 9.52 & 9.66 & 9.81 \\
$\left(\mathbf{m}^{\mathbf{2}} \mathbf{~ m i n}^{-1}\right)$ & & & \\
$\mathbf{h} \mathbf{x} \mathbf{~ 1 0}^{-6}$ & 0.93 & 1.32 & 2.33 \\
$\left(\mathbf{m} \mathbf{~ m i n}^{-1}\right)$ & 2.40 & 6.12 & 1.90 \\
Número de Biot & 0.9989 & 0.9969 & 0.9979 \\
$\mathbf{R}^{\mathbf{2}}$ & 2.3510 & 3.8607 & 8.8456 \\
$\chi^{2} \mathbf{x} \mathbf{~ 1 0}^{-3}$ & &
\end{tabular}

Nota: $\mathrm{R}^{2}$ - Coeficiente de determinação; $\chi^{2}$ - função qui-quadrado. Fonte: Própria (2021).

Os valores da difusividade efetiva de massa variaram de 9.52 a $9.81 \times 10^{-11}\left(\mathrm{~m}^{2} \mathrm{~min}^{-1}\right)$ quando a temperatura de secagem variou de 45 a $55^{\circ} \mathrm{C}$, visto que quando a temperatura é elevada a viscosidade da água diminuiu, facilitando assim a sua remoção. Segundo Santos et al. (2019b) a difusividade corresponde às magnitudes das taxas de secagem afetadas pela temperatura de secagem. Assim, as maiores taxas de secagem apresentaram os maiores valores de difusividade (Lang et al. 2018).

Os valores do coeficiente convectivo de transferência de calor tiveram uma tendência a proporcionalidade direta segundo o teste de correlação (+0.9688) com o aumento da temperatura de secagem variando de 0.93 a 2.33 x $10^{-6}\left(\mathrm{~m} \mathrm{~min}{ }^{-1}\right)$. Podendo afirmar que dentre as temperaturas aplicadas, a temperatura de $55^{\circ} \mathrm{C}$ possibilitou maior quantidade de calor transferida para o produto. 
Observa-se que o número de Biot variou de 1.90 a 6.12 não apresentando relação direta com o aumento da temperatura de secagem. Segundo Giner et al. (2010) e Bezerra et al. (2015), o número de Biot é um número adimensional que correlaciona a taxa de condução interna com a taxa de convecção externa e é capaz de indicar a resistência interna do produto ao processo de transferência de calor e massa. Portanto, cabe ressaltar que, segundo Silva et al. (2012) e Moreira et al. (2018), para um número tão baixo de Biot, a série infinita que representa a solução da equação de difusão pode ser representada apenas pelo seu primeiro mandato, com erro insignificante de truncamento.

Na Tabela 4 são mostrados os resultados para a caracterização físico-química e dos compostos bioativos dos pós obtidos nas temperaturas de 45,50 e $55^{\circ} \mathrm{C}$.

Tabela 4. Resultado da caracterização físico-química e dos compostos bioativos dos pós da casca de jabuticaba.

\begin{tabular}{|c|c|c|c|}
\hline \multirow{2}{*}{ Parâmetros } & \multicolumn{3}{|c|}{ Temperaturas } \\
\hline & $45^{\circ} \mathrm{C}$ & $50^{\circ} \mathbf{C}$ & $55^{\circ} \mathrm{C}$ \\
\hline Rendimento (\%) & $10.64 \pm 0.11^{\mathrm{A}}$ & $10.19 \pm 0.09^{\mathrm{B}}$ & $10.04 \pm 0.05^{\mathrm{B}}$ \\
\hline Atividade de água $\left(a_{w}\right)$ & $0.33 \pm 0.01^{\mathrm{A}}$ & $0.34 \pm 0.01^{\mathrm{A}}$ & $0.33 \pm 0.01^{\mathrm{A}}$ \\
\hline Teor de água \% (b.u.) & $9.66 \pm 0.13^{\mathrm{A}}$ & $9.33 \pm 0.78^{\mathrm{A}}$ & $9.15 \pm 0.98^{\mathrm{A}}$ \\
\hline Luminosidade $(* \mathbf{L})$ & $15.60 \pm 0.03^{\mathrm{C}}$ & $15.79 \pm 0.05^{\mathrm{B}}$ & $21.96 \pm 0.06^{\mathrm{A}}$ \\
\hline Intensidade de vermelho $\left(*_{a}\right)$ & $3.34 \pm 0,01^{\mathrm{B}}$ & $6.68 \pm 0.07^{\mathrm{B}}$ & $9.28 \pm 0.13^{\mathrm{A}}$ \\
\hline Intensidade de azul $(* b)$ & $5.81 \pm 0.01^{\mathrm{A}}$ & $4.47 \pm 0.11^{\mathrm{B}}$ & $5.42 \pm 0.17^{\mathrm{C}}$ \\
\hline $\begin{array}{c}\text { Compostos fenólicos totais } \\
\left(\text { mg GAE } 100 \mathrm{~g}^{-1}\right)\end{array}$ & $3546.77 \pm 5.77^{\mathrm{A}}$ & $3144.96 \pm 6.21^{\mathrm{B}}$ & $2801.62 \pm 4.15^{\mathrm{C}}$ \\
\hline Antocianinas totais $\left(\mathrm{mg} 100 \mathrm{~g}^{-1}\right)$ & $97.13 \pm 0.71^{\mathrm{A}}$ & $95.35 \pm 0.13^{\mathrm{A}}$ & $91.87 \pm 1.03^{\mathrm{B}}$ \\
\hline Flavonoides totais $\left(\mathrm{mg}^{100 \mathrm{~g}^{-1}}\right)$ & $143.81 \pm 1.03^{\mathrm{A}}$ & $114.48 \pm 0.52^{\mathrm{B}}$ & $83.19 \pm 0.75^{\mathrm{C}}$ \\
\hline
\end{tabular}

Nota: GAE (Equivalente de Ácido Gálico). Fonte: Própria (2021).

O rendimento na produção do pó em relação as cascas de jabuticaba úmidas variaram de 10.04 a 10.64\% em relação as temperaturas de secagem $\left(45,50\right.$ e $\left.55^{\circ} \mathrm{C}\right)$, onde o maior rendimento foi encontrado para a temperatura de $45^{\circ} \mathrm{C}$.

Verifica-se através da Tabela 4, que o processo de secagem não acarretou diferenças significativas entre para os parâmetros de atividade de água e teor de água, provavelmente pode-se atribuir aos intervalos de temperatura utilizados. Para a atividade de água os valores obtidos estão abaixo de 0.6, condição segura para impossibilitar o desenvolvimento de microrganismos no produto (Chisté et al., 2006). Os teores de água apresentados estão dentro dos padrões exigidos para pós ou farinhas (Brasil, 2005).

Para a análise de cor dos pós obtidos da casca de jabuticaba, observa-se que ocorreram diferenças significativas em relação as temperaturas aplicadas, sendo a temperatura de $55^{\circ} \mathrm{C}$ a que apresentou aumento na luminosidade e nas cromaticidades, indicando que houve degradação das antocianinas responsáveis pela cor do produto, visto que estas substâncias são termosensíveis. Dentre os pós obtidos, o da temperatura de $50{ }^{\circ} \mathrm{C}$ revelou-se ser o que mais se aproximou da cor roxo-escura, atribuída a presença de antocianinas na casca da jabuticaba quando o fruto está completamente maduro. Segundo Wu e Sun (2013) a cor dos pós de ervas é um dos atributos mais importantes e que pode influenciar na escolha do consumidor.

Os teores de compostos bioativos dos pós das cascas de jabuticaba nas três temperaturas apresentaram diferenças significativas. Os valores de compostos fenólicos totais mostraram diminuição com o aumento de temperatura, evidenciando que os polifenóis são sensíveis ao calor. Dentre as três temperaturas, a de $45{ }^{\circ} \mathrm{C}$ foi a que mais preservou o conteúdo dos compostos 
fenólicos após processo de secagem. Comportamento semelhante foi encontrado por Alves et al. (2014a) onde verificaram que os valores de compostos fenólicos e antocianinas totais foram mais preservados na temperatura de $45^{\circ} \mathrm{C}$ e Lenquiste et al. (2015) quando trabalharam com a casca de jabuticaba liofilizada encontraram valor de $3612.00 \mathrm{mg}$ GAE $100 \mathrm{~g}^{-1}$. Frauches et al. (2016) encontraram valores inferiores usando a temperatura de $60^{\circ} \mathrm{C} \mathrm{em}$ secador convectivo (2149.58 mg GAE $100 \mathrm{~g}^{-1}$ ). Segundo Cruz et al. (2014), temperaturas e velocidades de secagem mais baixas, como as utilizadas no processo de secagem deste estudo, reduzem as perdas da atividade antioxidante e dos compostos fenólicos.

Foi possível observar que os maiores teores de antocianinas foram obtidos nas temperaturas de 45 e $50{ }^{\circ} \mathrm{C}$, e os de flavonoides para a temperatura de $45^{\circ} \mathrm{C}$. Valores próximos foram reportados por Rufino et al. (2010) ao avaliarem a casca de

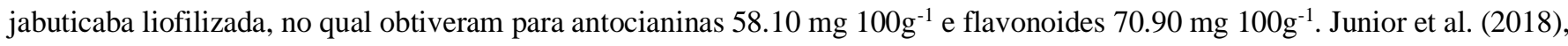
observaram que a maior degradação de antocianinas na casca de jabuticaba foi para a maior temperatura analisada $\left(70{ }^{\circ} \mathrm{C}\right)$. Sobrinho et al. (2010) afirmaram que a temperatura de secagem empregada não deve exceder a $60{ }^{\circ} \mathrm{C}$, pois temperaturas acima desta podem reduzir os níveis de flavonoides.

\section{Conclusão}

As cascas frescas de jabuticaba apresentaram altos teores de compostos fenólicos totais e taninos totais. O modelo de Midilli se destacou dos demais por apresentar o maior valor de $\mathrm{R}^{2}(>99 \%)$ e o menor valor da função qui-quadrado $(<0.000327)$ para as temperaturas estudadas, principalmente a de $45{ }^{\circ} \mathrm{C}$. A cinética de secagem das cascas de jabuticaba mostrou que a temperatura influenciou o processo, e que a constante de secagem " $\mathrm{k}$ " aumentou com a sua elevação, e o tempo do processo diminuiu em função do seu aumento. A temperatura de $45{ }^{\circ} \mathrm{C}$ garantiu uma maior preservação dos compostos fenólicos totais, antocianinas totais e flavonoides totais em relação as cascas de jabuticaba frescas. Logo, como o pó das cascas da jabuticaba seca tem uma quantidade significativa de compostos bioativos poderá ser uma alternativa como ingrediente na elaboração de vários produtos dentre eles: pão, bolo, biscoito, iogurte e bebida láctea.

\section{Referências}

AOAC. 2016. Official methods of analysis of AOAC International (20th ed.). Rockville, AOAC international.

Alezandro, M. R., Granato, D., \& Genovese, M. I. (2013). Jaboticaba (Myrciaria jaboticaba (Vell.) Berg), a Brazilian grape-like fruit, improves plasma lipid profile in streptozotocin-mediated oxidative stress in diabetic rats. Food Research International, 54(1), 650-659.

Alves, A. P. C., Corrêa, A. D., Alves, D. S., Saczk, A. A., Lino, J. B., \& Carvalho, G. A. (2014). Toxicity of the phenolic extract from jabuticabeira (Myrciaria cauliflora (Mart.) O. Berg) fruit skins on Spodoptera frugiperda. Chilean journal of agricultural research, 74(2), $200-204$.

Batista, Â. G., da Silva-Maia, J. K., Mendonça, M. C. P., Soares, E. S., Lima, G. C., Junior, S. B., ... \& Júnior, M. R. M. (2018). Jaboticaba berry peel intake increases short chain fatty acids production and prevent hepatic steatosis in mice fed high-fat diet. Journal of Functional Foods, $48,266-274$.

Bezerra, C. V., Meller da Silva, L. H., Corrêa, D. F., \& Rodrigues, A. M. C. (2015). A modeling study for moisture diffusivities and moisture transfer coefficients in drying of passion fruit peel. International Journal of Heat and Mass Transfer, 85, 750-755.

Brasil. (2005). Inclusão do uso das espécies vegetais e parte (s) de espécies vegetais para o preparo de chás. A Resolução de Diretoria Colegiada. Agência Nacional de Vigilância Sanitária, foi editada em complementação as espécies aprovadas pela RDC n ${ }^{\circ} 267$, de 22 de setembro de 2005.

Brasil. Instituto Adolfo Lutz. (2008). Métodos físico-químicos para análise de alimentos. (4a ed.), Digital, p. 1020.

Chang, S., Tang, H., Wu, H., Su, X., Lewis, A., \& Ji, C. (2018). Three-Dimensional Modelling and Simulation of the Ice Accretion Process on Aircraft Wings. International Journal of Astronautics and Aeronautical Engineering.

Chisté, R. C., Cohen, K. D. O., Mathias, E. D. A., \& Ramoa Júnior, A. G. A. (2006). Qualidade da farinha de mandioca do grupo seca. Food Science and Technology, 26(4), 861-864.

Coman, V., Teleky, B. E., Mitrea, L., Martau, G. A., Szabo, K., Calinoiu, L. F., \& Vodnar, D. C. (2019). Bioactive potential of fruit and vegetable wastes. Advances in Food and Nutrition Research.

Costa, C. F., Corrêa, P. C., Vanegas, J. D., Baptestini, F. M., Campos, R. C., \& Fernandes, L. S. (2016). Mathematical modeling and determination of thermodynamic properties of jabuticaba peel during the drying process. Revista Brasileira de Engenharia Agrícola e Ambiental, 20(6), 576-580. 
Cruz, S. K., De Moura, C. M., Meireles, G. C., da Silveira, C. M., \& Duarte, L. S. (2017). Secagem de bagaço de oliveira: influência da velocidade do ar na concentração de compostos bioativos. Anais do Salão Internacional de Ensino, Pesquisa e Extensão, 8(2).

Curi, P. N., Almeida, A. B. D., Pio, R., Lima, L. C. D. O., Nunes, C. A., \& Souza, V. R. D. (2019). Optimization of native Brazilian fruit jelly through desirabilitybased mixture design. Food Science and Technology, 39(2), 388-395.

Dessimoni-Pinto, N. A. V., Moreira, W. A., Cardoso, L. D. M., \& Pantoja, L. A. (2011). Jaboticaba peel for jelly preparation: an alternative technology. Food Science and Technology, 31(4), 864-869.

Francis, F. J. (1982). Analysis of anthocyanins in foods. In: Markakis P, Anthocyanins as Food Colors. Academic Press, 181-207.

Frauches, N. S., do Amaral, T. O., Largueza, C. B. D., \& Teodoro, A. J. (2016). Brazilian myrtaceae fruits: a review of anticancer proprieties. Journal of Pharmaceutical Research International, 1-15.

Gastaldi, B., Marino, G., Assef, Y., Sofrás, F. S., Catalán, C. A. N., \& González, S. B. (2018). Nutraceutical properties of herbal infusions from six native plants of argentine Patagonia. Plant foods for human nutrition, 73(3), 180-188.

Giner, S. A., Irigoyen, R. M. T., Cicuttín, S., \& Fiorentini, C. (2010). The variable nature of Biot numbers in food drying. Journal of Food Engineering, 101(2), $214-222$.

Júnior, A. M. O., Santos, J. T. S., \& Souza, D. F. S. (2018). Evaluation of different drying models for jabuticaba peel, dried in spouted bed dryer through performance indicators and the impact on anthocyanins. Scientia Plena, 14(6).

Lang, G. H., Lindemann, I. D. S., Ferreira, C. D., Pohndorf, R. S., Vanier, N. L., \& de Oliveira, M. (2018). Influence of drying temperature on the structural and cooking quality properties of black rice. Cereal Chemistry, 95(4), 564-574.

Lenquiste, S. A., da Silva Marineli, R., Moraes, É. A., Dionísio, A. P., de Brito, E. S., \& Junior, M. R. M. (2015). Jaboticaba peel and jaboticaba peel aqueous extract shows in vitro and in vivo antioxidant properties in obesity model. Food Research International, 77, 162-170.

Lenquiste, S. A., de Almeida Lamas, C., da Silva Marineli, R., Moraes, É. A., Borck, P. C., Camargo, R. L., ... \& Junior, M. R. M. (2019). Jaboticaba peel powder and jaboticaba peel aqueous extract reduces obesity, insulin resistance and hepatic fat accumulation in rats. Food Research International, $120,880-887$.

Lima, A. D. J. B., Corrêa, A. D., Alves, A. P. C., Abreu, C. M. P., \& Dantas-Barros, A. M. (2008). Caracterização química do fruto jabuticaba (Myrciaria cauliflora Berg) e de suas frações. Archivos Latinoamericanos de Nutricion, 58(4), 416.

Lima, A. D. J. B., Correa, A. D., Saczk, A. A., Martins, M. P., \& Castilho, R. O. (2011). Anthocyanins, pigment stability and antioxidant activity in jabuticaba [Myrciaria cauliflora (Mart.) O. Berg]. Revista Brasileira de Fruticultura, 33(3), 877-887.

Luikov, A.V. (1968). Analytical Heat Diffusion Theory. Academic Press, Inc., Ltd., London.

Melo, J. C., Pereira, E. D., Oliveira, K. P., Costa, C. H., \& Feitosa, R. M. (2015). Estudo da cinética de secagem da pimenta de cheiro em diferentes temperaturas Study smell pepper drying kinetics in different temperature. Revista Verde (Pombal-PB-Brasil), 10(2), 09-14.

Morales-Soto, A., García-Salas, P., Rodríguez-Pérez, C., Jiménez-Sánchez, C., de la Luz Cádiz-Gurrea, M., Segura-Carretero, A., \& Fernández-Gutiérrez, A. (2014). Antioxidant capacity of 44 cultivars of fruits and vegetables grown in Andalusia (Spain). Food Research International, 58, 35-46.

Moreira, I. D. S., da Silva, W. P., de Castro, D. S., de Melo Silva, L. M., \& Gomes, J. P. (2018). Production of kiwi snack slice with different thickness: Drying kinetics, sensory and physicochemical analysis. Australian Journal of Crop Science, 12(5), 778

Nascimento, A. P. S., Barros, S. L., Santos, N. C., Araújo, A. J. B., Cavalcanti, A. S. R. R. M., \& Duarte, M. E. M. (2018). Secagem convectiva e influência da temperatura nas propriedades físico-químicas das amêndoas de girassol comercial. Revista Brasileira de Produtos Agroindustriais, 20(3), 227-238.

Pádua, H. C., da Silva, M. A. P., Souza, D. G., Moura, L. C., Plácido, G. R., Couto, G. V. L., \& Caliari, M. (2017). Iogurte sabor banana (Musa AAB, subgrupo prata) enriquecido com farinha da casca de jabuticaba (Myrciaria jabuticaba (Vell.) Berg.). Global Science and Technology, 10(1), 89-104.

Pansera, M. R., Santos, A. C., Paese, K., Wasum, R., Rossato, M., Rota, L. D., Pauletti, G.F., Serafini, L. A. (2003). Análise de taninos totais em plantas aromáticas e medicinais cultivadas no Nordeste do Rio Grande do Sul. Revista Brasileira de Farmacognosia, 13, 17-22.

Quatrin, A., Pauletto, R., Maurer, L. H., Minuzzi, N., Nichelle, S. M., Carvalho, J. F. C., ... \& Emanuelli, T. (2019). Characterization and quantification of tannins, flavonols, anthocyanins and matrix-bound polyphenols from jaboticaba fruit peel: A comparison between Myrciaria trunciflora and M. jaboticaba. Journal of Food Composition and Analysis, 78, 59-74.

Re, R., Pellegrini, N., Proteggente, A., Pannala, A., Yang, M., \& Rice-Evans, C. (1999). Antioxidant activity applying an improved ABTS radical cation decolorization assay. Free radical biology and medicine, 26(9-10), 1231-1237.

Rufino, M. S. M., Alves, R. E., Brito, E. S., Pérez-Jiménez, J., Sauracalixto, F., Mancini-Filho, J. (2010). Bioactive compounds and antioxidant capacities of 18 non-traditional tropical fruits from Brazil. Food Chemistry, 121, 996-1002

Rufino, M. S. M., Alves, R. E., De Brito, E. S., De Morais, S. M., Sampaio, C. D. G., Pérez-Jimenez, J., Saura-Calixto, F. D. (2007). Metodologia científica: determinação da atividade antioxidante total em frutas pela captura do radical livre ABTS•+. Embrapa Agroindústria Tropical-Comunicado Técnico (INFOTECA-E).

Santos, D. C., de Farias Leite, D. D., Lisboa, J. F., de Lima Ferreira, J. P., dos Santos, F. S., de Lima, T. L. B., \& da Costa, T. N. (2019a). Modelagem e propriedades termodinâmicas da secagem de fatias de acuri. Brazilian Journal of Food Technology, 22, 1-12.

Santos, F. S. D., de Figueirêdo, R. M., Queiroz, A. J. D. M., \& Santos, D. D. C. (2017). Cinética de secagem e caracterização física e química de cascas de pitaya com polpa branca. Revista Brasileira de Engenharia Agrícola e Ambiental, 21(12), 872-877. 
Research, Society and Development, v. 10, n. 3, e21510313214, 2021

(CC BY 4.0) | ISSN 2525-3409 | DOI: http://dx.doi.org/10.33448/rsd-v10i3.13214

Santos, N. C., Silva, W. P., Barros, S. L., Araújo, A. D. B., Gomes, J. P., Almeida, R. L. J., \& Figueirêdo, R. M. F. (2019b). Study on Drying of Black Rice (Oryza sativa L.) Grains: Physical-Chemical and Bioactive Quality. Journal of Agricultural Science, 11(9), 203-212.

Seraglio, S. K. T., Schulz, M., Nehring, P., Della Betta, F., Valese, A. C., Daguer, H., \& Costa, A. C. O. (2018). Nutritional and bioactive potential of Myrtaceae fruits during ripening. Food chemistry, 239, 649-656.

Silva, E. C. O., da Silva, W. P., Gomes, J. P., Silva, C. M. D. P. S., Alexandre, H. V., Farias, V. S. O., \& de Figuiredo, R. M. F. (2019). Drying of Albedo and Whole Peel of Yellow Passion Fruit. Journal of Agricultural Science, 11(6), 501-509.

Silva, L. M. R., De Figueiredo, E. A. T., Ricardo, N. M. P. S., Vieira, I. G. P., De Figueiredo, R. W., Brasil, I. M., \& Gomes, C. L. (2014). Quantification of bioactive compounds in pulps and by-products of tropical fruits from Brazil. Food chemistry, 143, 398-404.

Silva, W. P., Farias, V. S. O., Neves, G. A., Lima, A. G. B. (2012). Modeling of water transport in roof tiles by removal of moisture at isothermal conditions. Heat Mass Transf. 48, 809-821.

Silva, W. P., Precker, J. W., e Silva, C. M., \& Gomes, J. P. (2010a). Determination of effective diffusivity and convective mass transfer coefficient for cylindrical solids via analytical solution and inverse method: Application to the drying of rough rice. Journal of food Engineering, 98(3), 302-308.

Sobrinho, P., da Silva, T. J., Gomes, T. D. L. B., Cardoso, K. C. D. M., Amorim, E. L. C. D., \& Albuquerque, U. P. D. (2010). Otimização de metodologia analítica para o doseamento de flavonoides de Bauhinia cheilantha (Bongard) Steudel. Química Nova, 33(2), 288-291.

Stasoft. (2007). Statistica 7.0 for Windows - Computar program manual Tulsa: Statsoft, Inc, CD Room.

Waterhouse, A. (1999). Folin-ciocalteau micro method for total phenol in wine. American Journal of Enology and Viticulture, 1, 3-5.

Wu, D., \& Sun, D. W. (2013). Colour measurements by computer vision for food quality control-A review. Trends in Food Science \& Technology, 29(1), 5-20. Doi: 10.1016/j.tifs.2012.08.004

Wu, S. B., Long, C., \& Kennelly, E. J. (2013). Phytochemistry and health benefits of jaboticaba, an emerging fruit crop from Brazil. Food Research International, 54(1), 148-159. 